\section{Case-Control and}

\section{Cohort studies}

reporting biomarker

prevalence data

allowing calculatin of

population

attributable risk (PAR)
ADULT ASTHMA

\section{CASE-CONTROL (OR}

NESTED

CASE/CONTROL)

Hahn \& Golubjatnikov Poylvalent (IgM, $\quad \geq 1: 1$

(1994)

IgG, IgA) MIF

\section{3}

Cook et al. (1998)

IgM, IgG, IgA MIF IgG 64-256 or

$\lg A \geq 8$

$10.1136 /$ thx. 53.4 .254

$\geq 1: 16$

$\geq 1: 16$

68

$47 \quad 69.1 \%$

Hahn et al. (2000)

IgA MIF
Hahn et al. (2000) IgG MIF

89

47

$52.8 \%$

47.2\% Persistent chronic asthma outpatient

22.1\% Severe chronic asthma, outpatient
8.6\% AAWI: asthma associated with infection at onset of first reported symptoms

43.2\% 25.9\% AAWI: asthma associated with infection at onset of first reported symptoms 


\begin{tabular}{|c|c|c|c|c|c|c|c|c|c|c|}
\hline Gencay et al. (2001) & IgG MIF & $\geq 1: 32$ & 33 & 21 & $63.6 \%$ & 33 & 19 & $57.6 \%$ & $6.1 \%$ & $\begin{array}{l}\text { Asthma severity no } \\
\text { All cases and contro } \\
\text { smokers. }\end{array}$ \\
\hline Gencay et al. (2001) & IgA MIF & $\geq 1: 20$ & 33 & 17 & $51.5 \%$ & 33 & 9 & $27.3 \%$ & $24.2 \%$ & $\begin{array}{l}\text { Asthma severity no } \\
\text { All cases and contro } \\
\text { smokers. }\end{array}$ \\
\hline Gencay et al. (2001) & IgG MIF & $\lg (\geq 1: 512$ & 33 & 6 & $18.2 \%$ & 33 & 1 & $3.0 \%$ & $15.2 \%$ & $\begin{array}{l}\text { Asthma severity no } \\
\text { All cases and contro } \\
\text { smokers. }\end{array}$ \\
\hline Gencay et al. (2001) & IgG \& IgA MIF & $\begin{array}{l}\lg G \geq 1: 512 \\
\text { and } \lg A \geq 1: 20\end{array}$ & 33 & 6 & $18.2 \%$ & 33 & 1 & $3.0 \%$ & $15.2 \%$ & $\begin{array}{l}\text { Asthma severity no } \\
\text { All cases and contro } \\
\text { smokers. }\end{array}$ \\
\hline \multicolumn{11}{|c|}{ 10.1164/ajrccm.163.5.2003162 } \\
\hline $\begin{array}{l}\text { Foschino Barbaro et } \\
\text { al. (2002) }\end{array}$ & IgG by MIF & $\lg G \geq 1: 16$ & 197 & 60 & $30.5 \%$ & 185 & 57 & $30.8 \%$ & $-0.4 \%$ & $\begin{array}{l}\text { Only } 17(8.6 \%) \text { of } 1 \\
\text { cases were severe } \\
\text { controls were hospi } \\
\text { general community }\end{array}$ \\
\hline \multicolumn{11}{|c|}{ 10.1046/j.1469-0691.2002.00430.x. } \\
\hline von Hertzen et al. & IgG MIF & $\geq 1: 256$ & 49 & 11 & $22.4 \%$ & 50 & 12 & $24.0 \%$ & $-1.6 \%$ & Mild asthma \\
\hline von Hertzen et al. & IgG MIF & $\geq 1: 256$ & 54 & 20 & $37.0 \%$ & 50 & 12 & $24.0 \%$ & $13.0 \%$ & Moderate asthma \\
\hline von Hertzen et al. & IgG MIF & $\geq 1: 256$ & 13 & 5 & $38.5 \%$ & 50 & 12 & $24.0 \%$ & $14.5 \%$ & Severe asthma \\
\hline von Hertzen et al. & IgG MIF & $\geq 1: 256$ & 116 & 36 & $31.0 \%$ & 150 & 36 & $24.0 \%$ & $7.0 \%$ & TOTAL \\
\hline von Hertzen et al. & IgA MIF & $\geq 320$ & 49 & 13 & $26.5 \%$ & 50 & 8 & $16.0 \%$ & $10.5 \%$ & Mild asthma \\
\hline von Hertzen et al. & IgA MIF & $\geq 320$ & 54 & 25 & $46.3 \%$ & 50 & 8 & $16.0 \%$ & $30.3 \%$ & Moderate asthma \\
\hline von Hertzen et al. & IgA MIF & $\geq 320$ & 13 & 6 & $46.2 \%$ & 50 & 8 & $16.0 \%$ & $30.2 \%$ & Severe asthma \\
\hline von Hertzen et al. & IgA MIF & $\geq 320$ & 116 & 44 & $37.9 \%$ & 150 & 24 & $16.0 \%$ & $21.9 \%$ & TOTAL \\
\hline
\end{tabular}


Biscione et al. (2004)

$$
\begin{array}{ll}
\text { nested RT-PCR on } & \text { any + nasal } \\
\text { MOMP mRNA } & \text { secretion } \\
& \text { sampled q2w } \\
& \text { for } 3 \text { months }
\end{array}
$$

$\underline{10.1183 / 09031936.04 .00049004}$

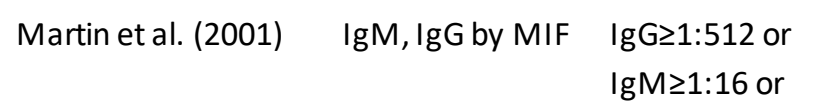

10.1067/mai.2001.113563

$\begin{array}{lll}\text { Tuuminen et al. } & \text { IgG by EIA } & >45 \\ & \text { IgA by EIA } & >12\end{array}$

10.1111/j.1198-743X.2004.00822.x

$\begin{aligned} \text { Hahn et al. (1996) } & \text { IgG by MIF } \\ \text { IgA by MIF } & \geq 1: 10\end{aligned}$

$\mathbf{0 . 0 \%}$ Asthma severity not specified. 6.7\% Asthma severity not specified.

$10.1017 / \mathrm{s} 0950268800059197$

\section{$-\mathbf{1 . 3 \%}$ The majority of cases were} atopic, severity not specified; all controls were non-atopic. Lower than expected populstion prevalence. 


\section{Routes et al. (2000) IgG by MIF}

$\geq 1: 64$

46

29

$63.0 \%$

46

30

$65.2 \%$

10.1016/s0091-6749(00)90093-9

$\begin{array}{ll}\text { IgG by MIF } & \geq 1: 32 \\ \text { IgG by MIF } & \geq 1: 64 \\ \text { IgA by MIF } & \geq 1: 16\end{array}$

15

$60.0 \%$

28

25

$11 \quad 44.0 \%$

28

25

$4 \quad 16.0 \%$

28

$10.1007 / \mathrm{s} 15010-003-2130-9$

Park et al. (2005)

$\lg M, \lg G$ by MI

$\lg \mathrm{g}>1 ; 512$ or

36

$\lg M>1: 20$

200504225 [pii]

Rodriguez et al. (2005) IgG by ELISA

against Cp LPS

\section{6}

Harju et al. (2006)

Cp PCR of nasal

swabs and/or

Harju et al. (2006)

P PCR of nasal

swabs and/or

$10.1136 /$ thx. 2005.056291
OD $>1.1$

55

13

$23.6 \%$

87

9

$10.3 \%$

13.3\% Asthma severity not specified.

Intrinsic asthma.
$50.0 \% \quad \mathbf{1 0 . 0} \%$ Mild late-onset non-atopic asthmatic non-smokers.

0.0\% $\quad \mathbf{4 4 . 0 \%}$ Mild late-onset non-atopic asthmatic non-smokers.

0.0\% $\quad \mathbf{1 6 . 0 \%}$ Mild late-onset non-atopic asthmatic non-smokers.

\section{$2.2 \% \quad 6.1 \%$ No biomarker data for lesser amounts of seroreactivity provided.}


Paldanius et al. (2007) IgG by MIF Paldanius et al. (2007) IgA by MIF

\section{$\lg G \geq 1: 32$}

$\lg A \geq 1: 10$

10.1016/j.diagmicrobio.2007.04.004

Cp IgG by MIF

$\lg \mathrm{g} \geq 1: 16$

84

53

$63.1 \%$

34

PCR of throat

wash

\section{$10.1080 / 02770900701815735$}

Torshizi et al. (2008) Culture of NP

$07.01 /$ ijaai.4546

Dejsomritrutai et al. IgG by MIF

(2009)

Dejsomritrutai et al.

IgA by MIF

(2009)

\section{3}

Specjalski et al. (2011) IgG by ELISA
Seropositivity perSavyon
1.4\% Nested case-control study from a population-based cohort. Asthma severity not specified. Did not require currently symptomatic asthma.

$\begin{array}{llllll}\lg A \geq 1: 32 & 52 & 15 & 28.8 \% & 137 & 30\end{array}$

$21.9 \%$

6.9\% Nested case-control study from a population-based cohort. Asthma severity not specified. Did not require currently symptomatic asthma.
21
$36.2 \% \quad 24.8 \% 25(26.3 \%)$ controlled, 23 (24.2\%) partly controlled, 47 (49.5\%) uncontrolled.




\begin{tabular}{|c|c|c|c|c|c|c|c|c|c|}
\hline IgG by ELISA & Seropositivity & 25 & 10 & $40.0 \%$ & 58 & 21 & $36.2 \%$ & $3.8 \%$ & controlled \\
\hline IgG by ELISA & Seropositivity & 23 & 15 & $65.2 \%$ & 58 & 21 & $36.2 \%$ & $29.0 \%$ & partly controlled \\
\hline IgG by ELISA & Seropositivity & 47 & 33 & $70.2 \%$ & 58 & 21 & $36.2 \%$ & $34.0 \%$ & uncontrolled \\
\hline IgA by ELISA & $\begin{array}{l}\text { Seropositivity } \\
\text { per Savyon }\end{array}$ & 95 & 42 & $44.2 \%$ & 58 & 17 & $29.3 \%$ & $14.9 \%$ & $\begin{array}{l}25(26.3 \%) \text { controlled, } 23 \\
(24.2 \%) \text { partly controlled, } 47 \\
(49.5 \%) \text { uncontrolled. }\end{array}$ \\
\hline
\end{tabular}

$\begin{array}{lllllllll}\text { IgA by ELISA } & \text { Seropositivity } & 25 & 3 & 12.0 \% & 58 & 17 & \mathbf{2 9 . 3 \%} & \mathbf{- 1 7 . 3 \%} \text { controlled } \\ \text { IgA by ELISA } & \text { Seropositivity } & 23 & 11 & 47.8 \% & 58 & 17 & \mathbf{2 9 . 3 \%} & \mathbf{1 8 . 5 \%} \text { partly controlled } \\ \text { IgA by ELISA } & \text { Seropositivity } & 47 & 28 & 59.6 \% & 58 & 17 & \mathbf{2 9 . 3 \%} & \mathbf{3 0 . 3 \%} \text { uncontrolled }\end{array}$

10.2500/aap.2011.32.3431

\begin{abstract}
Hahn et al. (2012) Cp-specific IgE by immunoblotting

Hahn et al. (2012) Cp-specific IgE by immunoblotting

Hahn et al. (2012) Cp-specific IgE by immunoblotting

Hahn et al. (2012) Cp-specific IgE by immunoblotting
\end{abstract}

10.1371/journal. pone.0035945

Smith-Norowitz et al. Cp-IgE by EIA (2020)

10.1016/j.heliyon.2020.e03512
$O D \geq 0.464$

22

$2195.5 \%$

22

10
7.8\% 24.2\% Mild intermittent and persistent asthma

7.8\% $\quad$ 44.0\% Moderate persistent asthma

7.8\% $\quad$ 70.7\% Severe persistent asthma

7.8\% $\quad$ 42.2\% TOTAL

\section{COHORT}




\begin{tabular}{|c|c|}
\hline $\begin{array}{l}\text { Pasternak et al. } \\
\text { (2005) }\end{array}$ & Cp IgG by MIF \\
\hline Pasternak et al. & Cp IgA by Mif \\
\hline \multicolumn{2}{|c|}{ 10.1016/j.jaci.2005.08.030 } \\
\hline PEDIATRIC ASTHMA & $\begin{array}{l}\text { Peripheral blood } \\
\text { unless otherwise }\end{array}$ \\
\hline
\end{tabular}

\section{CASE-CONTROL}

$\begin{array}{ll}\text { Emre 1995 } & \begin{array}{l}\text { IgE by } \\ \text { immunoblotting }\end{array} \\ \text { Emre 1995 } & \begin{array}{l}\text { IgE by } \\ \text { immunoblotting } \\ \text { IgE by } \\ \text { Emre 1995 }\end{array} \\ \text { immunoblotting } \\ \text { IgE by } 1995 \\ \text { Immunoblotting } \\ \text { Emre 1995 } & \begin{array}{l}\text { IgE by } \\ \text { immunoblotting }\end{array} \\ \text { Emre 1995 } & \begin{array}{l}\text { IgE by } \\ \text { immunoblotting } \\ \text { IgE by } \\ \text { Emre 1995 }\end{array} \\ \text { immunoblotting } \\ \text { I0.1093/infdis/172.1.265 }\end{array}$

Mills et al. (2000) IgG MIF 


\begin{abstract}
Nagy et al. (2003)
IgG by ELISA
IgA by ELISA

10.1016/s0091-6749(03)02010-4

Webley et al. (2005) Blood culture for $\mathrm{Cp}$
\end{abstract}

$\geq 1.1$

$\geq 1.1$

\subsection{4/rccm.200407-917OC}

Kopriva et al. (2005) IgM, IgG, IgA by $\begin{array}{ll}\text { ELISA } & \text { or IgG and } \\ \lg A, \text { cutoff }\end{array}$

10.5507/bp.2005.044

Teig et al. (2005)

PCR on nasal

brushings and/or

$10.1136 /$ thx. 2005.041004

Dal Molin et al. (2005) IgG by MIF

Criterion for seropositivity not presented

Dal Molin et al. (2005) IgA by MIF

Criterion for seropositivity not presented
42 $17 \quad 40.5 \%$ 70 66 $43.9 \%$ 241

26

$4 \quad 15.4 \%$

42

0

0.0\% $\quad \mathbf{1 5 . 4 \%}$ Asthma severity not reported.
$8 \quad 17.0 \%$

602

180

(18)

123
$5 \quad 10.6 \%$

602
29.9\% -12.9\% Asthma was by self-report. Control group did not exclude subjects with rrespiratory ailments. Asthma severity not reported.

20.4\% $\quad-\mathbf{9 . 8 \%}$ Asthma was by self-report. Control group did not exclude subjects with rrespiratory ailments. Asthma severity not reported. 
Annagur et al. (2007) IgG by ELISA

$10.1136 / j c p .2004 .024380$

$\begin{array}{ll}\text { Wazir et al. (2007) } & \text { IgG by ELISA } \\ \text { Wazir et al. (2007) } & \text { IgG by ELISA } \\ \text { Wazir et al. (2007) } & \text { IgG by ELISA } \\ \text { Wazir et al. (2007) } & \text { IgG by ELISA } \\ \mathbf{1 7 3 5 1 3 0 5} & \end{array}$

$\begin{array}{ll}\text { Nagy et al. (2007) } & \text { IgG by ELISA } \\ \text { Nagy et al. (2007) } & \text { IgA by ELISA }\end{array}$

10.2500/aap.2007.28.2957

\begin{abstract}
Kazar et al. (2011)
IgG by ELISA

$O D \geq 1.1$
\end{abstract}

97

$19 \quad 19.6 \%$

97

Seropositivity

Kazar et al. (2011) IgA by ELISA

$O D \geq 1.1$

$14 \quad 14.4 \%$
Criterion for seropositivity

not presented

$17 \quad 40.5 \%$

Criterion for seropositivity

Criterion for

seropositivity

Criterion for

seropositivity

Criterion for

seropositivity

Seropositivity

perSavyon,

perSavyon,
$45 \quad 31.9 \%$

187

90

61

$32.6 \%$

(1)
11.8\% $\quad \mathbf{1 9 . 0 \%}$ Mild asthma

11.8\% 38.2\% Moderate asthma

11.8\% 38.2\% Severe asthma

11.8\% $\quad \mathbf{2 9 . 0 \%}$ TOTAL

129

26

18
$20.2 \%$

$-0.6 \% 46$ mild intermittent, 40 mild persistent, 11 moderate persistent asthma

$\mathbf{1 4 . 0 \%} \quad \mathbf{0 . 5 \%} 46$ mild intermittent, 40 mild persistent, 11 moderate persistent asthma 
$10.1007 / \mathrm{s} 12223-011-0021-5$

Patel et al. (2012) Cp-specific lgE by
immunoblotting

of serum

10.1186/1465-9921-13-32

Tutanç et al.

Cp IgG by indirect Titre $\geq 1 / 100$

immunofluoresce

nce

10.4328/JCAM.2052
$54.8 \%$ lung diseases, unresponsive to corticosteroids; 143 had severe refractory asthma. Results for asthma subgroup noy reported separately.

13.0\% 14.2\% Positive association with more frequent attacks, but overall severity not specified 